\title{
Petrov types D and II perfect-fluid solutions in generalized Kerr-Schild form
}

\author{
F. Martín-Pascual and J. M. M. Senovillaa) \\ Departamento de Fisica Térica, Universidad de Salamanca, 37008, Salamanca, Spain
}

(Received 24 April 1986; accepted for publication 2 December 1987)

Petrov types D and II perfect-fluid solutions are obtained starting from conformally flat perfect-fluid metrics and by using a generalized Kerr-Schild ansatz. Most of the Petrov type D metrics obtained have the property that the velocity of the fluid does not lie in the two-space defined by the principal null directions of the Weyl tensor. The properties of the perfect-fluid sources are studied. Finally, a detailed analysis of a new class of spherically symmetric static perfect-fluid metrics is given.

\section{INTRODUCTION}

In a previous paper, ${ }^{1}$ the first results concerning perfectfluid solutions of Einstein's equations in generalized KerrSchild form were given. The generalized Kerr-Schild metrics have the following form:

$$
\tilde{g}_{\alpha \beta}=g_{\alpha \beta}+2 H l_{\alpha} l_{\beta},
$$

where $g_{\alpha \beta}$ is the metric of any space-time, $H$ is a scalar field, and $l_{\alpha}$ is a null geodesic vector field for both metrics $g$ and $\tilde{g}$.

As is well known, the Kerr-Schild ansatz has been a powerful tool in searching for solutions of Einstein's equations in either the vacuum case or the Einstein-Maxwell case. ${ }^{2-6}$ In contrast, excepting a metric due to Vaidya, ${ }^{7}$ no perfect-fluid solutions in generalized Kerr-Schild form were known up to the appearance of Ref. 1. In our opinion, there were two reasons for that lack. First, it was usual to start from metrics $g$ which were solutions of the vacuum Einstein equations. But, if $T_{\alpha \beta}$ and $\widetilde{T}_{\alpha \beta}$ are the energy-momentum tensors for the metrics $g$ and $\tilde{g}$, respectively, it was shown in Ref. 1 that the following interesting relation holds ${ }^{8}$ :

$$
l^{\alpha} \widetilde{T}_{\alpha \beta}=l^{\alpha} T_{\alpha \beta}+f l_{\beta},
$$

where $f$ is a scalar field. Therefore, if $T_{\alpha \beta}$ vanishes then $l_{\alpha}$ is a null eigenvector of $\widetilde{T}_{\alpha \beta}$, so that $\widetilde{T}_{\alpha \beta}$ cannot be the energymomentum tensor of a perfect fluid. The same happens when $l_{\alpha}$ is an eigenvector of $T_{\alpha \beta}$. Thus in order to obtain perfectfluid metrics $\tilde{g}$ we must consider only the case in which $l_{\alpha}$ is not a null eigenvector of $T_{\alpha \beta}$. Of course, the most interesting case arises when $T_{\alpha \beta}$ itself is an energy-momentum tensor for a perfect fluid.

The second reason emerges from the fact that it is necessary to allow great freedom in choosing the vector field $l^{\alpha}$. For example, in the classical Kerr-Schild metrics (Ref. 2), the great variety of shear-free null geodesic vector fields in flat space-time was used. For any metric $g$, there will be, in general, a great number of null geodesic vector fields. ${ }^{9}$ But, in order to solve the Einstein equations, it is also very useful to know an explicit expression of the general solution for vector fields of this kind. In the classical Kerr-Schild metrics it was very useful that the Kerr theorem ${ }^{10}$ provides the general solution for the shear-free geodesic null vector fields in flat space-time explicitly. This is not the case for an arbi-

\footnotetext{
a) Present address: Theoretical Astronomy Unit, School of Mathematical Sciences, Queen Mary College, Mile End Road, London E1 4NS, England.
}

trary metric $g$. However, it is known that the geodesic (shear-free) null vector fields in a conformally flat spacetime are the geodesic (shear-free) null vector fields in flat space-time, and conversely. Thus if we start from a conformally flat space-time then we can use the Kerr theorem. Moreover, the conformally flat perfect-fluid metrics have an additional advantage: all metrics of this kind are known. They are either generalized interior Schwarzschild solutions or generalized Friedmann solutions. ${ }^{11}$

Therefore, we shall start from conformally flat perfectfluid metrics $g$. We devote Sec. II to writing down the Einstein equations for this case. The case when the vector field $l^{\alpha}$ is shearing was studied in Ref. 1 . On the other hand, the case when $l^{\alpha}$ is shear-free was solved only in a very particular subcase. In this paper, we try to solve the shear-free case in general. There are two outstanding subcases which are studied in Sec. III.

All the solutions we obtain are Petrov types D and II. We also point out that most of the type-D metrics are new since the velocity of the fluid does not lie in the two-space spanned by the two multiple null eigenvectors of the Weyl tensor. Apart from the results obtained in Ref. 1, only two metrics (Wahlquist, ${ }^{12} \mathrm{Kramer}^{13}$ ) with this property were known previously.

In Sec. IV we give some explicit examples. In Ref. 1 two explicit examples of how the method works and their respective metrics were given. Although the method always works in the same way, in this paper we present some explicit solutions again. In particular, a class of spherically symmetric static perfect-fluid metrics is obtained. The properties of the perfect-fluid sources themselves are discussed in Sec. V. Finally, Sec. VI is devoted to the study of the new class of spherically symmetric static perfect-fluid space-times.

\section{THE EINSTEIN EQUATIONS}

Hereafter, we choose the metric $g$ and the null vector field $l$ of (1) with the following properties. First, $g$ is a solution of Einstein's equations for a perfect-fluid energy-momentum tensor,

$$
\begin{aligned}
& R_{\alpha \beta}=\chi\left(T_{\alpha \beta}-\frac{1}{2} g_{\alpha \beta} T\right), \\
& T_{\alpha \beta}=(q+p) u_{\alpha} u_{\beta}+p g_{\alpha \beta}, \quad g^{\alpha \beta} u_{\alpha} u_{\beta}=-1 .
\end{aligned}
$$

Second, $g$ is conformally flat, that is to say

$$
C_{\alpha \beta \lambda \mu}=0 \text {, }
$$


where $C_{\alpha \beta \lambda \mu}$ is the Weyl tensor for the metric $g$. Or, equivalently, $g$ can be transformed into

$$
g_{\alpha \beta} d x^{\alpha} d x^{\beta}=2 \phi^{2}(-d u d v+d z d \bar{z}),
$$

where $\phi^{2}$ is a positive function of the coordinates (the conformal factor) and where suitable coordinates $\{u, v, z, \bar{z}\}$ have been chosen for the flat metric. Finally, we choose the null geodesic vector field $l_{\alpha}$ to be shear-free. The general solution for vector fields of this kind in the metric (6) is known and is given by ${ }^{10,11}$ (Kerr theorem)

$$
l_{\alpha} d x^{\alpha}=d u+\bar{Y} d z+Y d \bar{z}+Y \bar{Y} d v,
$$

where $Y$ is a complex function of the coordinates defined implicitly by

$$
F(Y, \bar{z} Y+u, v Y+z)=0
$$

and where $F$ is an arbitrary analytic function of three complex variables.

Now, we choose a null tetrad $\{l, k, m, \bar{m}\}$ associated with $l$ as follows:

$$
m=m_{0}+\bar{Z} l, \quad k=k_{0}+Z m_{0}=\bar{Z} \bar{m}_{0}+Z \bar{Z} l,
$$

where

$$
m_{0}=\phi(d z+Y d v), \quad k_{0}=\phi^{2} d v
$$

and

$$
\bar{Z}=-m_{0} \cdot u / l \cdot u \text {. }
$$

Therefore, in this null tetrad we have

$$
m^{\alpha} u_{\alpha}=0
$$

or, equivalently, ${ }^{14}$

$$
\begin{aligned}
& \phi_{01}=\phi_{02}=\phi_{12}=0, \quad \phi_{00}=(\chi / 2)(q+p)\left(l^{\alpha} u_{\alpha}\right)^{2}, \\
& \phi_{11}=(\chi / 8)(q+p), \quad \phi_{22}=(\chi / 8)(q+p)\left(l^{\alpha} u_{\alpha}\right)^{-2}, \\
& \Lambda=(\chi / 24)(q-3 p) .
\end{aligned}
$$

The spin coefficients ${ }^{14}$ for the null tetrad (9) are given in Refs. 1 and 15 explicitly. Here, we only remark that

$$
\varkappa=\epsilon=\sigma=\lambda=\beta=0 .
$$

If we are looking for solutions $\tilde{g}$ for a perfect-fluid energymomentum tensor

$$
\widetilde{T}_{\alpha \beta}=(\tilde{q}+\tilde{p}) \tilde{u}_{\alpha} \tilde{u}_{\beta}+\tilde{p} \tilde{g}_{\alpha \beta}, \quad \tilde{g}^{\alpha \beta} \tilde{u}_{\alpha} \tilde{u}_{\beta}=-1,
$$

and if we define

$$
U \equiv \delta H-2 H \bar{\alpha}, \quad V \equiv D H-2 H \rho,
$$

the Einstein equations become finally ${ }^{1}$ ( $\chi$ is the gravitational constant)

$$
\begin{aligned}
& \chi \tilde{p}=\chi p-D V-V(3 \rho+\bar{\rho})-3 H \rho^{2}-4 H \rho \bar{\rho} \\
& +H\left(\bar{\rho}^{2}+4 \phi_{00}\right), \\
& \chi \tilde{q}=\chi q+V(\bar{\rho}-\rho)-D V+3 H\left(\rho^{2}+\bar{\rho}^{2}\right), \\
& \left(l^{\alpha} \tilde{u}_{\alpha}\right)^{2}=2 \phi_{00}\left\{\chi(q+p)+4 H \phi_{00}\right. \\
& \quad-2[D V+2 V \rho+2 H \bar{\rho}(\rho-\bar{\rho})]\}^{-1}, \\
& m^{\alpha} \tilde{u}_{\alpha}=0, \\
& \delta V+(\rho+\bar{\rho}) U+(\tau-\bar{\alpha}) V \\
& +H[\bar{\rho} \bar{\alpha}+\delta \bar{\rho}+2 \tau(\bar{\rho}-\rho)]=0
\end{aligned}
$$

$$
\begin{aligned}
\rho \Delta H= & -H \Delta(\rho+\bar{\rho})-\delta \bar{U}+\bar{\alpha} \bar{U}-\bar{\tau} U-\tau \bar{U}-\mu V \\
& -4 H \phi_{11}+H(\gamma+\bar{\gamma})(\rho-\bar{\rho})-W,
\end{aligned}
$$

where we have put

$$
\begin{aligned}
W \equiv & \left(1 / 4 \phi_{00}\right)[D V+2 V \rho+2 H \bar{\rho}(\rho-\bar{\rho})] \\
& \times\left[D V+2 V \rho+2 H \bar{\rho}(\rho-\bar{\rho})-4 H \phi_{00}-8 \phi_{11}\right] .
\end{aligned}
$$

The expressions (17)-(19) are not equations, but they define $\tilde{q}, \tilde{p}$, and $\tilde{u}^{\alpha}$ as functions of $q, p, u^{\alpha}$, and the unknown $H$. Thus we need only solve Eqs. (20) and (21), which are differential equations for $H$. However, in addition to Eqs. (20) and (21) we have the compatibility conditions for $U$ and $V$, that is, ${ }^{1,15}$

$$
\begin{aligned}
& D U+(2 \bar{\rho}-\rho) U+(\tau+\bar{\pi}) V \\
& \quad+H[\bar{\rho} \bar{\alpha}+\delta \bar{\rho}+2 \pi(\rho-\bar{\rho})]=0, \\
& \bar{\delta} U+\bar{\rho} \Delta H+\bar{\mu} \bar{V}-U \alpha+2 H \rho(\gamma+\bar{\gamma})=\text { c.c. }
\end{aligned}
$$

Also, from the definition (16) of $V$ it follows that

$$
V-\bar{V}=2 H(\bar{\rho}-\rho) \text {. }
$$

The Weyl tensor for the metric $\tilde{g}$ is given by ${ }^{16}$

$$
\begin{aligned}
& \tilde{\psi}_{0}=\tilde{\psi}_{1}=0,-6 \tilde{\psi}_{2}=[D-2(\rho-\bar{\rho})] V, \\
& \tilde{\psi}_{3}=(2 \rho-\bar{\rho}) \bar{U}+H[\rho \alpha+\bar{\delta} \rho+\bar{\tau}(\rho-\bar{\rho})], \\
& \bar{\psi}_{4}=-[\bar{\delta}-(3 \alpha-2 \bar{\tau})] \bar{U} .
\end{aligned}
$$

Therefore, all the metrics $\tilde{g}$ are algebraically special and the vector field $l^{\alpha}$ is a multiple null eigenvector of the Weyl tensor.

When $V=0$, from (23), (25), and the Newman-Penrose equations, it follows that $U=0$ and $\rho=\bar{\rho}$. Then, from (26) it is evident that $\tilde{g}$ is conformally flat. Thus we shall only consider the case $V \neq 0$.

It has become clear to the authors that cases which have a function $W$ nonlinear in $H$ do not have solutions in general. This is because $W$ is always of greater order in $H$ than $V$ and $U$. And then, Eqs. (16) and (21) are not compatible in general. ${ }^{1,15}$ Perhaps, this fact has something to do with a theorem due to Xanthopoulos (see Refs. 5 and 6). Moreover, it is convenient to assume that

$$
\rho=\bar{\rho} .
$$

This assumption simplifies the calculations substantially. Therefore, we shall treat the following two cases:

$$
\begin{array}{ll}
\text { case A, } & D V+2 V \rho=0, \\
\text { case B, } & D V+2 V \rho-4 H \phi_{00}=0 .
\end{array}
$$

Of course, there are more cases in which $W$ becomes linear in $H$, for example, when $D V+2 V \rho-8 \phi_{11}=0$ and $D V+2 V \rho-4 H \phi_{00}-8 \phi_{11}=0$. These two cases would provide different resulting metrics, which is a proof of the variety of possibilities in our Kerr-Schild transformation. However, all the cases are formally equivalent to either the case $A$ or the case $B$, and then the calculations are just a pure repetition in other cases. We shall study both cases in the following section.

Because of (13) and (27) we can use the Bianchi identities for the metric $g$ as given in the Appendix of Ref. 1. These identities and the Newman-Penrose equations for the metric 
$g$ (when they are conveniently restricted to each case) will be used repeatedly (but not explicitly) in Sec. III. Anyway, we shall omit the details.

\section{THE SOLUTIONS}

First, we assume the condition (28). From this assumption and (27), Eqs. (20), (21), (23), (24), and (25) become, respectively,

$\delta V+2 \rho U+(\tau-\bar{\alpha}) V=0$,

$\rho \Delta H=-2 H \Delta \rho-\delta \bar{U}+\bar{\alpha} \bar{U}-\bar{\tau} U-\mu V-4 H \phi_{11}$,

$D U+\rho U+(\tau+\bar{\pi}) V=0$,

$\bar{\delta} U-U \alpha=\delta \bar{U}-\bar{U} \bar{\alpha}$,

$V=\bar{V}$.

The compatibility of (30) with (28), making use of (32), leads us to

$$
\left(\rho^{2}-\phi_{00}\right) U=\tau \rho V .
$$

We can consider two cases.

(A1) $\rho^{2}-\phi_{00} \neq 0$. Then Eq. (35) tells us that

$U=\tau \rho V /\left(\rho^{2}-\phi_{00}\right)$. have

(A2) $\rho^{2}-\phi_{00}=0$. Then Eq. (35) implies that we must

$$
\tau=0
$$

In case (A1), from (28) and (32) and by applying the operator $D$ to $\left(35^{\prime}\right)$ we obtain

$$
\begin{aligned}
& \pi=0, \\
& \tau\left[\rho D \phi_{00}+2 \phi_{00}\left(\rho^{2}+\phi_{00}\right)\right]=0 .
\end{aligned}
$$

Therefore, we can consider two subcases again.

(A1a) $\rho D \phi_{00}=-2 \phi_{00}\left(\rho^{2}+\phi_{00}\right)$. From the Newman-Penrose equations and the Bianchi identities it is easily shown that this condition is equivalent to

$$
\phi_{00}=C \rho^{2} \text {, }
$$

where $C$ is an arbitrary positive real constant. In order to distinguish this case from the case (A2) we must assume that

$$
C \neq 1 \text {. }
$$

(A1b) $\tau=0$. In this case (37) is automatically satisfied. Furthermore, this case is different from the case (A2) since now we have $\rho^{2}-\phi_{00} \neq 0$.

Next, we are going to solve the three subcases (A1a), (A1b), and (A2) separately.

The subcase (Ala): From the above considerations, Eqs. (30) and (28) are already compatible and Eq. (32) is satisfied. Also, Eq. (31) now becomes

$$
\begin{aligned}
\Delta \mathbf{H}= & 2 H(\mu+\gamma+\bar{\gamma})-\mu V / \rho+2 V\left(\mu \rho+\phi_{11}\right. \\
& +\Lambda) / \rho^{2}(1-C)+2 V \tau \bar{\tau} / \rho^{2}(1-C)^{2} .
\end{aligned}
$$

The compatibilities of this equation with $V$ and $U$ give us, respectively,

$$
\begin{aligned}
\Delta V= & \mu V(2-C)-\frac{2 V \Lambda}{\rho}+(\gamma+\bar{\gamma}) V-\frac{4 \phi_{11} V}{\rho} \\
& -\frac{2 \tau \bar{\tau} V(3-C)}{\rho(1-C)^{2}}-\frac{4 V\left(\mu \rho+\phi_{11}+\Lambda\right)}{\rho(1-C)},
\end{aligned}
$$

$$
\begin{aligned}
\Delta \tau= & \mu \tau(2+C)-(\gamma-\bar{\gamma}) \tau \\
& +\frac{2 \tau \Lambda}{\rho}-\frac{2 \tau\left(\mu \rho+\phi_{11}+\Lambda\right)}{\rho(1-C)} \\
& -\frac{2 \tau \phi_{11}(1-2 C)}{\rho C}-\frac{2 \bar{\tau} \tau^{2}}{\rho(1-C)} .
\end{aligned}
$$

Equation (41) is compatible with (28) and (30). Finally, a new integrability condition arises from (42) and the Newman-Penrose equations,

$$
(1+C) \mu \rho+2 \Lambda+2(2 C-1) \phi_{11} / C=0 .
$$

It is easily shown that condition (43) is possible.

The subcase (Alb): Now, we assume

$$
\tau=0, \quad \rho^{2} \neq \phi_{00}
$$

so that $\left(35^{\prime}\right)$ tells us that

$$
U=0 \text {. }
$$

Thus Eqs. (28) and (30) are compatible and Eqs. (32) and (33) are automatically satisfied. Moreover, Eq. (31) becomes

$$
\rho \Delta H=2 H \rho(\gamma+\bar{\gamma}-\mu)-4 H\left(\phi_{11}+\Lambda\right)-\mu V,
$$

which is compatible with (45). The compatibility of (46) with $V$ leads us to

$$
\begin{aligned}
\Delta V= & V(2 \mu+\gamma+\bar{\gamma})-2 V\left(2 \phi_{11}+\Lambda\right) / \rho-\mu \phi_{00} V / \rho^{2} \\
& -4 H \phi_{00}\left(\mu \rho+\phi_{11}+\Lambda\right) / \rho^{2} .
\end{aligned}
$$

Also, from (44) and (45) Eq. (30) becomes

$$
\delta V=\bar{\alpha} V .
$$

Equations (47) and (48) are compatible and the integrability of (47) and (28) gives us the following condition:

$$
\begin{aligned}
& V\left\{2 \phi_{11}+\frac{\phi_{00}\left(7 \mu \rho+8 \phi_{11}+4 \Lambda\right)}{\rho^{2}}\right. \\
& \left.\quad+\frac{2 \mu \phi_{00}{ }^{2}}{\rho^{3}}+\frac{1}{\rho}\left[2 D \phi_{11}+\frac{\mu}{\rho} D \phi_{00}\right]\right\} \\
& \quad+4 \frac{H}{\rho^{2}}\left(\mu \rho+\phi_{11}+\Lambda\right)\left(D \phi_{00}+4 \rho \phi_{00}+\frac{2 \phi_{00}{ }^{2}}{\rho}\right)=0 .
\end{aligned}
$$

It is very difficult to know if the expression (49) is possible in general. In fact, Eq. (49) should be interpreted as an equation from which $V$ is obtained as a function of $H$. Then we should put this $V$ in Eqs. (28), (47), and (48) and we should obtain an (or more!) expression for $H$ which is not, in general, a solution of Eqs. (16) and (46). This procedure is useless in general. However, we can assume

$$
\mu \rho+\phi_{11}+\Lambda=0
$$

so that Eq. (49) becomes

$$
\begin{aligned}
2 \phi_{11} & +\frac{\phi_{00}\left(\phi_{11}-3 \Lambda\right)}{\rho^{2}} \\
& +\frac{2 \mu \phi_{00}^{2}}{\rho^{3}}+\frac{1}{\rho}\left[2 D \phi_{11}+\frac{\mu}{\rho} D \phi_{00}\right]=0 .
\end{aligned}
$$

This is a condition on only $g$ and therefore we only have to check it. In fact, it may be shown that (51) is possible. tions

The subcase (A2): This case is defined by the assump- 


$$
\tau=0, \quad \phi_{00}=\rho^{2}
$$

so that Eqs. (28) and (30) are compatible. Furthermore, Eq. (31) may be written

$$
\begin{aligned}
\rho \Delta H= & 2 H\left[\rho(\gamma+\bar{\gamma})-\mu \rho-2\left(\phi_{11}+\Lambda\right)\right] \\
& -\mu V-\bar{\delta} U+\alpha U .
\end{aligned}
$$

The compatibility of this equation with $V$ gives us

$$
\begin{aligned}
\Delta V= & 2(\bar{\delta} U-\alpha U)-4 H\left(\mu \rho+\phi_{11}+\Lambda\right)+(V / \rho) \\
& \times\left[\rho(\mu+\gamma+\bar{\gamma})-2\left(2 \phi_{11}+\Lambda\right)\right] .
\end{aligned}
$$

In order to make this expression compatible with (28) we must have

$$
\mu \rho+\phi_{11}+\Lambda=0
$$

and then, Eqs. (53) and (54) become, respectively,

$$
\begin{aligned}
\rho \Delta H= & 2 H \rho(\mu+\gamma+\bar{\gamma})-\mu V-\bar{\delta} U+\alpha U, \\
\Delta V=2(\bar{\delta} U-\alpha U)+(V / \rho) & \\
& \times\left[\rho(\mu+\gamma+\bar{\gamma})-2\left(2 \phi_{11}+\Lambda\right)\right] .
\end{aligned}
$$

The compatibility of (56) with $U$ is

$$
\begin{aligned}
& \rho \Delta U+\bar{\delta} \delta U-3 \bar{\alpha} \bar{\delta} U \\
& \quad+U[3 \alpha \bar{\alpha}-\rho(5 \mu+3 \bar{\gamma}+\gamma)]=0 .
\end{aligned}
$$

Keeping this equation in mind, Eqs. (57) and (30) are compatible. Now, the Weyl tensor is given by

$$
3 \tilde{\psi}_{2}=V \rho, \quad \tilde{\psi}_{3}=\bar{U} \rho, \quad \tilde{\psi}_{4}=-(\bar{\delta}-3 \alpha) \bar{U} .
$$

Since $V$ does not vanish, we only can obtain solutions of Petrov types D and II. For Petrov type-D solutions we must have

$$
\delta U=U(3 \bar{\alpha}-2 \rho U / V) .
$$

Otherwise, the solutions are Petrov type II. By using the condition (33), and after a little computation, it is easily shown that Eqs. (60), (58), and (32) are compatible.

Now, we are going to solve case $B$. Therefore, we assume conditions (29) and (27) so that Eqs. (20), (23), (24), and (25) become, respectively, Eqs. (30), (32), (33), and (34). Furthermore, Eq. (21) now may be written

$$
\begin{aligned}
\rho \Delta H= & -2 H \Delta \rho-\delta \bar{U}+\bar{\alpha} \bar{U} \\
& -\bar{\tau} U-\tau \bar{U}-\mu V+4 H \phi_{11} .
\end{aligned}
$$

The compatibility of (30) with (29), making use of (32), leads us to (36) and (37). Also, we must have

$$
\left(\rho^{2}+\phi_{00}\right) U=\tau\left(V \rho-2 H \phi_{00}\right)
$$

Consequently, as in case $\mathrm{A}$, we could consider two subcases again but it turns out that the only interesting case arises when

$$
\tau=0
$$

and then, from (62) we have

$$
U=0 \text {. }
$$

Thus, Eqs. (29) and (30) are compatible and also Eq. (32) is satisfied. On the other hand, Eq. (61) becomes

$\rho \Delta H=2 H \rho(\gamma+\bar{\gamma}-\mu)+4 H\left(\phi_{11}-\Lambda\right)-\mu V$,

which is compatible with (64). The integrability of (65) and $V$ leads us to

$$
\begin{aligned}
\Delta V= & V(2 \mu+\gamma+\bar{\gamma})-2 \Lambda(V / \rho)-\mu V \phi_{00} / \rho^{2} \\
& +4 \phi_{11} V / \rho+4(H / \rho)\left[2 D \phi_{11}+2 \rho \phi_{11}\right. \\
& \left.+\left(\phi_{00} / \rho\right)\left(\phi_{11}-\Lambda-2 \mu \rho\right)\right] .
\end{aligned}
$$

This equation is compatible with (30). Moreover, it may be shown that under the conditions

$$
\begin{aligned}
& \mu \phi_{00}=2 \rho \phi_{11}, \\
& D \phi_{11}+\phi_{00}\left(\phi_{11}-\Lambda\right) / 2 \rho=0,
\end{aligned}
$$

Eq. (66) is compatible with (29). In fact, condition (67) is not very much restrictive because it is satisfied for the metrics $g$ which have a constant energy density. In other words, all the generalized interior Schwarzschild metrics satisfy the above-mentioned condition (67). ${ }^{11}$

The results obtained in this section can be summarized as follows.

Let us choose the conformally flat perfect-fluid metric $g$ and the shear-free geodesic null vector field $l^{\alpha}$ such that they verify the possible conditions given in the first row of Table $I$. Then, let us define $U$ and $V$ by (16) and let us solve the integrable system of equations for $U$ and $V$ which appear in the second row of the table. Once this has been done, the solutions $H$ of the compatible system of equations given by (16) and the third row of the table provide us generalized Kerr-Schild metrics $\tilde{g}$. These metrics are solutions of the Einstein equations for a perfect-fluid energy-momentum tensor (15), where the energy density $\tilde{q}$, the pressure $\tilde{p}$, and the velocity $\tilde{u}^{\alpha}$ are given, for each case, in the fourth row of the table. The Weyl tensor of the resulting metrics as well as their Petrov types are also shown in Table I.

As we can see in the table, the Petrov type-D metrics of cases A1a and A2 satisfy $\tilde{\psi}_{3} \neq 0$ and $\tilde{\psi}_{4} \neq 0$. Therefore, for these metrics, $\tilde{k}^{\alpha}$ is not a multiple null eigenvector of the Weyl tensor, and then the form of $\tilde{u}^{\alpha}$ tells us that the velocity of the fluid does not lie in the preferred two-space defined by the multiple null eigenvectors of the Weyl tensor. Thus these solutions are new. On the other hand, the metrics of cases $\mathrm{A} 1 \mathrm{~b}$ and $\mathrm{B}$ have $u^{\alpha}$ lying in that preferred two-space so that they may be already known. ${ }^{11}$

The particular case $V=C H \rho$ (where $C$ is a constant $\neq-2$ ) belongs to the more general case $\mathrm{A} 1 \mathrm{a}$ and it had been solved previously by one of us. ${ }^{17}$ Likewise, the case $V=-2 H \rho$ solved in Ref. 1 (when $\sigma=0$ ) belongs to the general case A2.

\section{EXPLICIT EXAMPLES}

In this section we give some examples of how the equations may be solved for each particular case.

(1) The most simple metric $g$ we can choose is the "flat" Robertson-Walker metric,

$d s^{2}=2 R^{2}(-d u d v+d z d \bar{z}), \quad R=R(t), \quad q=q(t)$,

$p=p(t), \quad t \equiv \frac{u+v}{2^{1 / 2}}, \quad \dot{q}=-\frac{3(q+p) \dot{R}}{R}$,

$\dot{R}^{2}=\frac{\chi}{3} q R^{4}, \quad u_{\alpha} d x^{\alpha}=-\frac{R(d u+d v)}{2^{1 / 2}}$,

$l_{\alpha} d x^{\alpha}=d u+\bar{Y} d z+Y d \bar{z}+Y \bar{Y} d v, \quad \dot{X} \equiv \frac{d X}{d t}$. 
TABLE I. Integrability conditions, compatible systems of equations, and properties for Kerr-Schild metrics.

\begin{tabular}{|c|c|c|c|c|}
\hline Case & Ala & Alb & A2 & $\mathbf{B}$ \\
\hline Condition on $g$ and $l$ & $(27),(36),(38),(42)$ & $(27),(44),(50),(51)$ & $(27),(52),(55)$ & $(27),(63),(67),(68)$ \\
\hline Equations for $U$ and $V$ & $\left(35^{\prime}\right),(28),(30),(41)$ & $(45),(28),(47),(48)$ & $(32),(33),(58),(28),(30),(57)$ & $(64),(29),(66),(30)$ \\
\hline Equation for $\Delta H$ & (40) & (46) & (56) & $(65)$ \\
\hline$\tilde{q}, \tilde{p}$, and $\tilde{u}^{\alpha}$ & & $(17)-(19)$ with (27), (28) & & $\begin{array}{c}(17)-(19) \\
\text { with }(27),(29)\end{array}$ \\
\hline Weyl tensor & $\begin{array}{c}3 \tilde{\psi}_{2}=V \rho \\
\tilde{\psi}_{3}=\bar{\tau} V /(1-C) \\
\tilde{\psi}_{4}=2 \bar{\tau}^{2} V / \rho(1-C)^{2}\end{array}$ & $\begin{aligned} 3 \tilde{\psi}_{2} & =V \rho \\
\tilde{\psi}_{3} & =0 \\
\tilde{\psi}_{4} & =0\end{aligned}$ & (59) & $\begin{array}{c}3 \tilde{\psi}_{2}=V \rho-2 H \phi_{\infty 0} \\
\tilde{\psi}_{3}=0 \\
\tilde{\psi}_{4}=0\end{array}$ \\
\hline Petrov & D & D & II or D & $\mathbf{D}$ \\
\hline
\end{tabular}

We try to solve the equations for the case A2. Thus we must restrict the metric $g$ and the vector field $l^{\alpha}$ such that conditions (27), (52), and (55) are satisfied. It is easily shown that these conditions are verified if and only if ${ }^{18}$

$$
Y=0, \quad q+3 p=0 .
$$

Then, the Robertson-Walker metric must be restricted such that

$$
\begin{aligned}
& q=(A / R)^{2}, \quad R=B e^{ \pm C t}, \\
& C \equiv(\chi / 3)^{1 / 2} A, \quad A, B=\text { const. }
\end{aligned}
$$

Equations (32), (33), and (58) for $U$ and Eqs. (28), (30), and (57) for $V$ leads us to

$$
U=f(z, \bar{z}, u) / e^{ \pm C t}, \quad V=G(z, \bar{z}, u) / e^{ \pm 2 C t},
$$

where the functions $f$ and $G$ verify the following equations:

$$
\begin{aligned}
& \frac{\partial f}{\partial z}=\text { c.c., } \quad \frac{\partial G}{\partial u} B=-2 \frac{\partial f}{\partial z}, \\
& \frac{\partial G}{\partial \bar{z}} B= \pm 2^{1 / 2} C f, \quad-2^{1 / 2} \frac{\partial^{2} f}{\partial z \partial \bar{z}}= \pm C \frac{\partial f}{\partial u} .
\end{aligned}
$$

A particular solution of these equations is given by

$$
B G= \pm 2^{1 / 2} C[M(\bar{z})+\bar{M}(z)], \quad f=\frac{\partial M}{\partial \bar{z}},
$$

where $M$ is an arbitrary complex function of the variable $\bar{z}$. Now, we know that the system of equations (56) and (16) for $H$ is compatible. The integration of this system is standard and we obtain for $H$

$$
H=B[M(\bar{z})+\bar{M}(z)]+E e^{ \pm 2 C t},
$$

where $E$ is an arbitrary constant. These metrics belong to the class of generalized Robinson-Trautman solutions. ${ }^{11,19} \mathrm{Un}$ less we have $M=$ const, the resulting metrics $\tilde{g}$ are Petrov type II.

Another particular solution of Eqs. (69) is given by

$$
\begin{gathered}
G B=-2 a \exp \left\{\mp C(u+z+\bar{z}) / 2^{1 / 2}\right\}=-2 f, \\
a=\text { const, }
\end{gathered}
$$

and then, the solution of Eqs. (56) and (16) for $H$ is

$$
C H=\mp 2^{1 / 2} a B \exp \left\{\mp C(u+z+\bar{z}) / 2^{1 / 2}\right\}+E e^{ \pm 2 C t} \text {. }
$$

In this case, Eq. (60) is also satisfied and therefore the resulting metrics $\tilde{g}$ are Petrov type D. Since $\tilde{\psi}_{3}$ and $\tilde{\psi}_{4}$ do not vanish they are new.
(2) In this example we take the interior Schwarzschild metric in canonical coordinates, that is to say ${ }^{11}$

$d s^{2}=-(A r)^{2} d t^{2}+N^{-2} d r^{2}+r^{2}\left(d \theta^{2}+\sin ^{2} \theta d \phi^{2}\right)$,

$A r=a-b N, \quad N^{2}=1-(r / R)^{2}, \quad a, b, R=$ const,

$\chi q=3 / R^{2}, \quad \chi p=(3 b N-a) / R^{2} A r$,

$u_{\alpha} d x^{\alpha}=-A r d t$,

and we try solve the equations for the case B. It may be shown that the only shear-free geodesic null vector field which satisfies $\tau=0,(27)$ and (68) is given by

$$
l_{\alpha} d x^{\alpha}=M(-d t+d r / A r N),
$$

where

$$
\begin{aligned}
M \equiv & 2^{1 / 2} A(1+\cos \theta)(A+B \sin \omega t+\omega \cos \omega t) \\
& \times[\sin \theta(A \sin \omega t+B)]^{-2}, \\
\mathrm{Br} \equiv & a N-b, \quad \omega^{2}=A^{2}-B^{2}=\left(a^{2}-b^{2}\right) / R^{2} .
\end{aligned}
$$

A null tetrad associated with $l_{\alpha}$ and such that $m^{\alpha} u_{\alpha}=0$ is given by ${ }^{20}$

$$
\begin{aligned}
8^{1 / 2} k_{\alpha} d x^{\alpha}= & r(1-\cos \theta)(\omega \cos \omega t-A-B \sin \omega t) \\
& \times(A r d t+d r / N), \\
2^{1 / 2} m_{\alpha} d x^{\alpha}= & r e^{i \phi}(-d \theta+i \sin \theta d \phi),
\end{aligned}
$$

so that the only non-null spin coefficients are

$$
\begin{aligned}
\rho= & M N / A r^{2}, \quad \mu=A N / 2 M, \\
\alpha= & -(1+\cos \theta) e^{-i \phi / 2^{1 / 2}} r \sin \theta, \\
\gamma= & \left(\frac{1}{8}\right)^{1 / 2}(1-\cos \theta) \\
& \times\left[N \omega \cos \omega t+\left(r / R^{2}\right)(a \sin \omega t-b)\right] .
\end{aligned}
$$

Solving the system of equations defined by (29), (30), (66), (16), and (65) for $V$ and $H$ we obtain

$$
V=\frac{A}{M}\left[\frac{N}{2} r f^{\prime}-\frac{B}{A} f\right], \quad 2 H=\left(\frac{A r}{M}\right)^{2} f,
$$

where $f(r)$ is a solution of the following differential equation:

$$
A r^{3} N^{2} f^{\prime \prime}+\left(r^{3} / R^{2}\right)(4 b N-a) f^{\prime}-2 A r f=0 .
$$

The resulting metric $\tilde{g}$ is a Petrov type-D static spherically symmetric perfect-fluid solution. In the following sections, 
we are going to discuss the properties of the solutions we have obtained.

\section{PROPERTIES OF THE SOLUTIONS}

It is evident that the properties of the generalized KerrSchild metrics $\tilde{g}$ depend, in general, on the properties of the initial metrics $g$ themselves. However, some considerations may be made without loss of generality and then the specific properties of the explicit solutions can be deduced.

Thus, for example, in Ref. 1 it was shown that Petrov type-N metrics cannot be obtained by means of the KerrSchild transformation as defined by us. Also, the Petrov type of the resulting metrics has been always given in Sec. III. It is convenient to remark that this has been possible because we knew the Petrov type of the initial metrics (they are conformally flat).

With regard to the symmetries of the solutions, one of us ${ }^{15}$ has shown the following result: " $\mathrm{S}$ is a Killing vector field of the Kerr-Schild metric $\tilde{g}$ if and only if

$$
£(\mathbf{S}) 1=f 1, \quad £(\mathbf{S}) g_{\alpha \beta}=-2[£(\mathbf{S}) H+2 H f] l_{\alpha} l_{\beta},
$$

where $f$ is a function of the coordinates and we use standard notation for Lie derivatives." This result provides us a method to find all the Killing vector fields of the explicitly known Kerr-Schild metrics. For the first solution of the previous section, the former conditions (82) lead us to

$$
\begin{aligned}
& \mathbf{S}= {\left[\left(A_{1}+\bar{A}_{1}\right) u+A_{3}\right] \frac{\partial}{\partial u} } \\
&-\left[\left(A_{1}+\bar{A}_{1}\right) u+A_{3} \pm \frac{A_{1}+\bar{A}_{1}}{2^{1 / 2} C}\right] \frac{\partial}{\partial v} \\
&+\left(A_{1} \bar{z}+A_{2}\right) \frac{\partial}{\partial \bar{z}}+\left(\bar{A}_{1} z+\bar{A}_{2}\right) \frac{\partial}{\partial z} \\
&\left(A_{1}+\bar{A}_{1}\right)\left(E+B^{2}\right)=0, \\
& 2\left(A_{1}+\bar{A}_{1}\right)(\mathbf{M}+\bar{M})+\left(A_{1} \bar{z}+A_{2}\right) \frac{d M}{d \bar{z}} \\
& \quad+\left(\bar{A}_{1} z+\bar{A}_{2}\right) \frac{d \bar{M}}{d z}=0,
\end{aligned}
$$

where $A_{1}, A_{2}$, and $A_{3}$ are arbitrary constants ( $A_{3}$ real). From these expressions it is evident that, in general, the only Killing vector is given by $A_{1}=A_{2}=0$, that is to say

$$
\mathbf{S}=\frac{\partial}{\partial u}-\frac{\partial}{\partial v}
$$

But also, there are some particular cases depending on the form of the function $\mathbf{M}(\bar{z})$. These are the following:

$$
\text { (a) If } \mathrm{M}(\bar{z})=c \bar{z}+d, \quad c, d=\text { const, } \quad c \neq 0 \text {, }
$$

then

$$
\mathbf{S}=i \bar{c} \frac{\partial}{\partial \bar{z}}-i c \frac{\partial}{\partial z}
$$

is another Killing vector.

(b) If $\mathbf{M}(\bar{z})=d$, then

$$
\frac{\partial}{\partial z}+\frac{\partial}{\partial \bar{z}}, \quad i\left(\frac{\partial}{\partial \bar{z}}-\frac{\partial}{\partial z}\right), \quad i\left[\bar{z} \frac{\partial}{\partial z}-z \frac{\partial}{\partial \bar{z}}\right]
$$

are Killing vectors as well. (c) If $\mathrm{M}(\bar{z})=(a / b) \log \left(i b \bar{z}+A_{2}\right), \quad a, b=$ real const, then

$$
\left(i b \bar{z}+A_{2}\right) \frac{\partial}{\partial \bar{z}}+\left(\bar{A}_{2}-i b z\right) \frac{\partial}{\partial z}
$$

is another Killing vector.

(d) If $M(\bar{z})=c\left(A_{1} \bar{z}+A_{2}\right)^{-2\left(1+\bar{A}_{\left.1 / A_{1}\right)}\right.}-i a /\left(A_{1}\right.$ $+\bar{A}_{1}$ ) and $E=-B^{2}$ then (83) with $A_{3}=0$ is a Killing vector. This is the only new Killing vector which is not a Killing vector of the initial metric $g$.

Similarly, the symmetries of the second solution of the previous section may be obtained. The result is that there are the following two Killing vectors:

$$
i\left(\frac{\partial}{\partial \bar{z}}-\frac{\partial}{\partial z}\right), \frac{\partial}{\partial u}-\frac{\partial}{\partial v}+\frac{1}{2}\left(\frac{\partial}{\partial \bar{z}}+\frac{\partial}{\partial z}\right) .
$$

Both of them were Killing vectors for the initial RobertsonWalker metric $g$.

In relation to the properties of the density and pressure of the solutions, first of all we must obtain the explicit expressions for these quantities, which are given by

$$
\chi \tilde{q}=I \exp (\mp 2 C t)+(M+\bar{M})\left(A^{2} / 3 B^{3}\right) \exp (\mp 4 C t) \text {, }
$$

$$
\begin{aligned}
\chi \tilde{p}= & -(I / 3) \exp (\mp 2 C t)+(M+\bar{M})\left(A^{2} / 3 B^{3}\right) \\
& \times \exp (\mp 4 C t),
\end{aligned}
$$

for the first metric obtained in Sec. IV, and by

$$
\begin{aligned}
\chi \tilde{q}= & I \exp (\mp 2 C t)+\left(2^{1 / 2} a A^{2} / 3 C B^{3}\right) \\
& \times \exp \left[\mp 4 C t \mp C(u+z+\bar{z}) / 2^{1 / 2}\right], \\
\chi \tilde{p}= & -(I / 3) \exp (\mp 2 C t)+\left(2^{1 / 2} a A^{2} / 3 C B^{3}\right) \\
& \times \exp \left[\mp 4 C t \mp C(u+z+\bar{z}) / 2^{1 / 2}\right],
\end{aligned}
$$

for the second metric, where

$$
I \equiv(A / B)^{2}\left(1+E / B^{2}\right) .
$$

From (85)-(88) it is clear that the solutions do not have singularities in general. Furthermore, both metrics satisfy

$$
\chi(\tilde{q}-\tilde{p})=(4 I / 3) \exp (\mp 2 C t) .
$$

Finally, we are going to study the properties of the velocity of the fluid of the Kerr-Schild metrics. By using the formulas of Ref. 21, making a change of null tetrad and after some standard and straightforward calculations we obtain for the shear, vorticity, and expansion of the fluid the following expressions:

$$
\begin{aligned}
& \sigma_{\alpha \beta}: A=-\left(\frac{1}{18}\right)^{1 / 2}\left\{(1+\epsilon) V+\frac{2 \phi_{11}}{\phi_{00} L^{2}} D H\right. \\
&-\frac{\epsilon}{L^{2}}[\Delta H-2 H(\gamma+\bar{\gamma})] \\
&-\frac{H}{L^{2} \phi_{00}}(1-\epsilon)\left[2 D \phi_{11}-\frac{2 \phi_{11}}{\phi_{00}} D \phi_{00}\right] \\
&\left.-\frac{2 H \epsilon}{L^{2} \phi_{00}}\left(2 \phi_{1} \rho-\mu \phi_{00}\right)\right\} L^{-1}, \\
& B=-\left(1 / 2 L^{2}\right)[H \bar{\tau}(1-\epsilon)+(1+\epsilon) \bar{U}] \\
& C=0, \\
& \omega_{\alpha \beta}: U=\left(1 / 2 L^{2}\right)(1-\epsilon)(H \bar{\tau}+\bar{U})
\end{aligned}
$$




$$
V=0
$$

$$
\begin{aligned}
\theta: \theta= & \frac{1}{2 L}\left\{(1+\epsilon)(D H+4 H \rho)+\frac{2 \phi_{11}}{\phi_{00} L^{2}} D H\right. \\
& -\frac{\epsilon}{L^{2}}[\Delta H-2 H(\gamma+\bar{\gamma})] \\
& -(1-\epsilon) \frac{H}{L^{2} \phi_{00}}\left[2 D \phi_{11}-\frac{2 \phi_{11}}{\phi_{00}} D \phi_{00}\right] \\
& \left.-\frac{\left(2 \phi_{1} \rho-\mu \phi_{00}\right)\left(\epsilon H / L^{2}-3\right)}{\phi_{00}}\right\},
\end{aligned}
$$

where we have used the notation of Ref. 21 and we have put

$$
L^{2}=2 \phi_{11} / \phi_{00}+\epsilon H \text {. }
$$

Moreover, $\epsilon=1$ for case A and $\epsilon=-1$ for case B. These expressions are valid in general. From (93) we see that the solutions obtained in this paper do not have vorticity. This is a direct consequence of assumption (27). On the other hand, they have, in general, shear, expansion, and acceleration. The explicit expressions of these quantities for the explicit metrics of Sec. IV may be easily obtained from (90)-(95). However, the static and spherically symmetric solution is shear-free and expansion-free (of course!). In the next section, we are going to study this particular solution.

\section{A CLASS OF STATIC, SPHERICALLY SYMMETRIC PERFECT-FLUID METRICS}

In Sec. IV, we obtained the metric

$$
\begin{aligned}
d \tilde{s}^{2}= & -(A r)^{2} d t^{2}+N^{-2} d r^{2}+r^{2}\left(d \theta^{2}+\sin ^{2} \theta d \phi^{2}\right) \\
& +(A r)^{2} f(r)(-d t+d r / A r N)^{2},
\end{aligned}
$$

where $f(r)$ is a solution of the differential equation (81). By making the following change of the timelike coordinate:

$$
d T=d t+[f / A r N(1-f)] d r,
$$

the metric (96) becomes

$$
\begin{aligned}
d \tilde{s}^{2}= & -(A r)^{2}(1-f) d T^{2}+\left[1 / N^{2}(1-f)\right] d r^{2} \\
& +r^{2}\left(d \theta^{2}+\sin ^{2} \theta d \phi^{2}\right),
\end{aligned}
$$

In this form, the metric is manifestly static and spherically symmetric. By using the formulas of previous sections we can get the velocity of the fluid

$$
\tilde{u}=A r(1-f)^{1 / 2} d T
$$

and the density and pressure

$$
\chi \tilde{q}=\chi q+\left(N^{2} / r\right) f^{\prime}+\left[1-3(r / R)^{2}\right] f / r^{2},
$$

$\chi \tilde{p}=\chi p-\left(N^{2} / r\right) f^{\prime}-\left[1-(3-2 a / A r)(r / R)^{2}\right] f / r^{2}$,

where $\chi q$ and $\chi p$ are the density and pressure of the Schwarzschild interior solution and are given in (72). By the way, we remark that the metric (98) is a generalization of the Schwarschild interior metric. The Schwarzschild metric is the particular case $f(r)=0$ [as is evident from (98)(101) or directly from $(80)]$.

If we want to study the properties of the solution (98) we have to solve the differential equation (81). This equation is linear and of second order and, in general, it has four regular singular points. ${ }^{22,23}$ This type of equation is called Heun's equation. ${ }^{22,23}$ However, there are two cases in which the equation has only three regular singular points (so that it can be reduced to the hypergeometric equation ${ }^{23}$ ). These cases are defined by $b=0$ and $b=a$. When $b=a$, it may be shown that there is not any regular solution. Then, we do not consider this case here.

Let us begin with the easier case $b=0$. In this case, (81) can be reduced to a hypergeometric equation and, in fact, the general solution of ( 81 ) may be expressed by means of elementary functions as follows:

$f(r)=3 C[1-(N R / r) \arcsin (r / R)]+c N R / r$,

where $C$ and $c$ are arbitrary constants. In order to have a regular solution in $r=0$ we should impose

$$
c=0 \text {. }
$$

Keeping this condition in mind, from (100) and (101) we get the density and pressure for this case,

$\chi \tilde{q}=\left(3 / R^{2}\right)[1-2 C+(3 C N R / r) \arcsin (r / R)]$,

$\chi \tilde{p}=-R^{-2}[1+(3 C N R / r) \arcsin (r / R)]$.

It follows from (104) and (105) that this solution satisfies the equation of state

$$
\chi(\tilde{q}+3 \tilde{p})=-6 C / R^{2}=\text { const. }
$$

By physical considerations, we must demand $C<0$ so that $\tilde{q}+3 \tilde{p}$ is positive and, also, this assures the correctness of the signature for $(98)$ because $f(r)<0$. This special metric is just the static limit of the Wahlquist solution ${ }^{12}$ and it was also given by Whittaker. ${ }^{24}$

Now, let us study the general case $b \neq 0$. By simplicity, it is convenient to distinguish several possibilities depending on the different values of $b / a$. Thus, for example, when $b / a \leqslant \frac{1}{3}$ the regular solution of $(81)$ is

$$
\begin{gathered}
f(r)=C(r / R)^{2} F[(b-a) / 2 b,(5 b-2 a) / b ; \\
\left.5,2, \frac{5}{2}, \frac{5}{2} ;(1-N) / 2\right],
\end{gathered}
$$

where $F$ is the solution of Heun's equation (see Ref. 22). The regular solution of (81) when $\frac{1}{3}<b / a<\frac{1}{2}$ is

$$
\begin{aligned}
f(r)= & C[2 b /(a-b)]^{3 / 2}(r / R)^{2} F \\
& \times\left[2 b /(b-a),(10 b-4 a) /(b-a) ; 5,2, \frac{5}{2}, 3 ; x\right],
\end{aligned}
$$

$x=[2 b(1-N) / 2(b-a)]$,

where $C$ is an arbitrary constant again. Analogously, the solution when $\frac{1}{2} \leqslant b / a<1$ may be given by means of the Heun's function $F$.

We shall restrict ourselves to the case $b / a \leqslant \frac{1}{3}$ because all the possibilities are quite similar. From (107) it may be shown

$f(0)=0, \quad \frac{f}{r^{2}}(r=0)=\frac{C}{R^{2}}, \quad \frac{f^{\prime}}{r}(r=0)=\frac{2 C}{R^{2}}$,

and then, the density and pressure are regular everywhere. Moreover, from (72), (100), (101), and (109), we have

$$
\begin{aligned}
& \chi \tilde{q}(0)=3(1+C) / R^{2}, \\
& \chi \tilde{p}(0)=R^{-2}[-3 C+(3 b-a) /(a-b)] .
\end{aligned}
$$

Therefore, we must choose $C$ as follows:

$$
-1<C<(3 b-a) / 3(a-b)
$$


so that $\tilde{\mathrm{q}}(0)$ and $\tilde{\mathrm{p}}(0)$ are positive.

Bearing this condition in mind, and taking into account the following relation:

$$
\chi \tilde{p}(r=R)=\chi p(r=R)=-R^{-2}<0,
$$

we conclude that the pressure is a decreasing function of $r$ and that there exists a value $r=r_{0}<R$ such that $\tilde{p}\left(r_{0}\right)=0$.

Unfortunately, it is very difficult to find out the equation of state for these metrics. However, from (100) and (101) it is evident that the following relation holds in general:

$$
\chi(\tilde{q}+\tilde{p})=\chi(q+p)(1-f)=\left[2 a / A r R^{2}\right](1-f) .
$$

This expression proves that there are no solutions in which the density and the pressure vanish at the same value of $r$ without singularities in the metric [see (98)] and, therefore, the equation of state cannot be that of a polytropic fluid.

'J. Martín and J. M. M. Senovilla, J. Math. Phys. 27, 265, 2209 (1986).

${ }^{2}$ G. C. Debney, R. P. Kerr, and A. Schild, J. Math. Phys. 10, 1842 (1969).

${ }^{3}$ A. Thompson, Tensor (N. S.) 17, 92 (1966).

${ }^{4} \mathrm{~J}$. Plebanski and A. Schild, Nuovo Cimento B 35, 35 (1976).

${ }^{5}$ A. H. Taub, Ann. Phys. (NY) 134, 326 (1981).

${ }^{6}$ B. C. Xanthopoulos, J. Math. Phys. 19, 1607 (1978); Ann. Phys. (NY) 149,286 (1983).

${ }^{7}$ P. C. Vaidya, Pramana 8, 512 (1977).

${ }^{8}$ Throughout the paper there are two kinds of objects related to the metrics $\tilde{g}$ and $g$. We denote these, respectively, by a tilde and by no label. Consequently, we raise and lower indices of the tensors with $\tilde{g}$ or $g$, respectively. It is easily shown that $\tilde{l}=l$ (see Ref. 1 ).
${ }^{9}$ However, when $l$ is also shear-free there is not, in general, enough freedom in choosing $l$ because of the generalized Goldberg-Sachs theorem (see Ref. 11).

${ }^{10}$ D. Cox and E. J. Flaherty, Jr., Commun. Math. Phys. 47, 75 (1976).

'D. Kramer, H. Stephani, M. MacCallum, and E. Herlt, Exact Solutions of Einstein's Field Equations (Deutscher Verlag der Wissenschaften, Berlin, 1980).

${ }^{12}$ H. D. Wahlquist, Phys. Rev. 172, 1291 (1968).

${ }^{13}$ D. Kramer, Class. Quantum Grav. 1, L3 (1984).

${ }^{14}$ Standard notation in Newman-Penrose formalism is used throughout the paper. Our conventions coincide with those of Kramer et al. (Ref. 11) except for the spin coefficients where the sign is changed.

${ }^{15}$ J. M. M. Senovilla, Ph.D. thesis, Universidad de Salamanca, 1986.

${ }^{16}$ The expressions (26) are calculated in the tetrad $\tilde{l}=l, \tilde{m}=m$, and $\tilde{k}^{\alpha}=k^{\alpha}+H l^{\alpha}$, (Ref. 1).

${ }^{17} \mathrm{~J}$. M. M. Senovilla, in Actes dels Encuentros Relativistas Españoles 85, Maó 1985, edited by A. Molina (L'E. T. S. E. I. B., Menorca, 1986).

${ }^{18}$ The metric $g$ does not have any physical meaning when we have obtained the new metric $g$. Then, we can allow the relationship $q+3 p=0$ since we only must impose physical restrictions to the new $\tilde{q}$ and $\tilde{p}$.

${ }^{19}$ J. Wainwright, Int. J. Theor. Phys. 10, 39 (1974).

${ }^{20}$ This null tetrad and the spin coefficients have been obtained by using the method developed in Sec. II (and Ref. 1) starting from the most general tetrad (7)-(11). Therefore, we have needed the conformal factor for the interior Schwarzschild metric, which is given by

$$
\phi=r(A \sin \omega t+B) \text {. }
$$

This explicit value has been found by J. Martín and J. M. M. Senovilla (unpublished) and as far as we know, it was unknown in this form up to now.

${ }^{21}$ J. Wainwright, J. Math. Phys. 18, 672 (1977). We use the formulas (A6)(A9) of Appendix A of this paper.

${ }^{22}$ E. Kamke, Differentialgleichungen Losungsmethoden und Losungen (Chelsea, New York, 1971).

${ }^{23}$ Bateman Manuscript Project, A. Erdélyi, W. Magnus, F. Oberhettinger, and F. G. Tricomi, Higher Transcendental Functions (McGraw-Hill, New York, 1953).

${ }^{24}$ J. M. Whittaker, Proc. R. Soc. London Ser. A 306, 1 (1968). 